\title{
Immunohistochemical demonstration of relaxin in the genital tract of men
}

\author{
Hannele Yki-Järvinen, T. Wahlström†, and M. Seppälä \\ Department I of Obstetrics and Gynecology, University Central Hospital, Helsinki and \\ †Department of Pathology, University of Helsinki, Finland
}

\begin{abstract}
Summary. The biotin-avidin immunoperoxidase staining method and antisera against highly purified porcine relaxin were utilized to localize relaxin in the male genital tract. Formalin-fixed tissue specimens from the prostate gland, the testis, epididymis, vas deferens and the seminal vesicle were studied. Specific relaxin immunoactivity was seen in the glandular epithelium of the prostate and also in the glandular epithelium of the seminal vesicle and in the ampullary part of the vas deferens. The testis and epididymis were negative.
\end{abstract}

\section{Introduction}

Human seminal plasma contains 7-40 times more relaxin than does serum from pregnant women when measured by a heterologous radioimmunoassay (RIA) for porcine relaxin (O'Byrne \& Steinetz, 1976; O’Byrne, Carriere, Sorensen, Segaloff \& Schwabe, 1978a; Quagliarello, Szlachter, Steinetz, Goldsmith \& Weiss, 1979; Loumaye, de Cooman \& Thomas, 1980). Human relaxin has been purified from the seminal plasma (Essig et al., 1981). The tissue origin of relaxin in men is, however, unknown. In ejaculates from men with congenital absence of the vas deferens the concentrations of relaxin measured with the porcine RIA are about 2 times that in normal men (Essig et al., 1981). This suggests that relaxin in the male may be produced by the prostate. Relaxin has been identified by an immunoperoxidase method in the armadillo prostate gland (Cameron, Corton \& Larkin, 1982). In the boar, relaxin was first reported to be present in the testis (Dubois \& Dacheux, 1978), but when antiserum against highly purified relaxin was used, specific fluorescence was not observed (Arakaki, Kleifeld \& Bryant-Greenwood, 1980). The purpose of the present study was to determine the tissue origin of relaxin in men.

\section{Materials and Methods}

Immunization. Highly purified porcine relaxin (NIH-RXN-P1, $3000 \mathrm{U} / \mathrm{mg}$ ) was obtained from the National Pituitary Agency, National Institute of Arthritis, Metabolic and Digestive Diseases (NIAMDD), NIH, Bethesda, Maryland, U.S.A. A New Zealand White male rabbit was immunized with $1 \mathrm{ml}$ emulsion containing $200 \mu \mathrm{g}$ NIH-RXN-P1 in $0.5 \mathrm{ml} 2 \mathrm{~mm}$-sodium phosphate, $\mathrm{pH} 7.5$, and $0.5 \mathrm{ml}$ Freund's complete adjuvant. Four s.c. injections were given dorsally. Booster injections containing $100 \mu \mathrm{g}$ relaxin were given every 3 weeks. The rabbit was bled 10 days after each booster injection, and serum was stored at $-20^{\circ} \mathrm{C}$ until tested. The immune sera (NIH-RXN-

* Reprint requests to Professor M. Seppälä, Department of Gynaecology and Obstetrics, University Central Hospital, Haartmaninkatu 2, Helsinki 29, Finland. 
P1-3000 antiserum) were tested by double diffusion against purified porcine relaxin, human prolactin (NIAMDD), human placental lactogen (Pharmacia, Uppsala, Sweden), human growth hormone (NIAMDD), porcine insulin (Novo, Copenhagen, Denmark), human chorionic gonadotrophin (hCG: the National Institute of Child Health and Human Development, NIH), pregnancy specific beta-1 glycoprotein (SP1: Behringwerke AG, Marburg, West Germany) and sera from pregnant women. Rabbit antiporcine relaxin antiserum R6 was kindly provided by $\mathrm{E}$. $\mathbf{M}$. O'Byrne (Ciba-Geigy Corporation, Ardsley, New York, U.S.A.). This antiserum was prepared against highly purified porcine relaxin isolated from pregnant sow ovaries (Sherwood \& O'Byrne, 1974) and inhibits the pubic symphysis-relaxing activity of extracts of human corpora lutea of pregnancy (Steinetz, O'Byrne, Weiss \& Schwabe, 1979). Human corpora lutea extracts and extracts of human pregnancy plasma yield concentration-dependent curves parallel to those obtained with porcine relaxin standards in two different RIA systems employing antiserum R6 (O'Byrne et al., 1978b; Loumaye, Teuwissen \& Thomas, 1978).

Tissue preparation. Tissue specimens from the prostate gland were obtained at surgery for benign hyperplasia of the prostate (10 patients). The tissues were fixed with $10 \%$ buffered $(0 \cdot 1 \mathrm{M}$ sodium phosphate $\mathrm{pH}$ 7.4) formalin and embedded in paraffin wax according to routine histological procedures. Haematoxylin-eosin-stained sections were used to differentiate between normal tissue and benign hyperplasia of the prostate. Only blocks containing light microscopically normal tissue were selected for immunoperoxidase staining. Samples of the testis, epididymis, vas deferens, seminal vesicle and prostate from 2 men were fixed (formalin) at autopsy within $1 \mathrm{~h}$ of traumatic death. Specimens from 5 retained testes were obtained at surgery.

Immunoperoxidase staining. Paraffin wax-embedded tissue was treated to remove the wax before being stained in the following manner. Endogenous peroxidase activity was destroyed by incubation with methanol containing $0.3 \%$ hydrogen peroxide at room temperature for $30 \mathrm{~min}$. After washing with phosphate-buffered saline (PBS) containing $2 \%$ normal rabbit serum, $\mathrm{pH} 7 \cdot 2$, the sections were incubated with NIH-RXN-P1-3000 antiserum or antiserum R6 diluted $1: 50$ and $1: 200$ respectively with $\mathrm{PBS}$ at $37^{\circ} \mathrm{C}$ for $60 \mathrm{~min}$. After washing with $\mathrm{PBS}$, the sections were incubated with biotinylated antirabbit IgG antibody solution (Biotinylated Antibody Solution in Vectastain ABC KIT, Vector Laboratories Inc., Burlingname, California, U.S.A.) at $37^{\circ} \mathrm{C}$ for 30 min, washed with PBS and incubated with avidin and horseradish peroxidase (Vectastain ABC Reagent) at $37^{\circ} \mathrm{C}$ for $30 \mathrm{~min}$. After washing again with PBS, the chromogenic reaction was developed by incubating with a solution containing $0.04 \%$ 3-amino-9-ethyl-carbazole and $0.01 \%$ hydrogen peroxide in $0.05 \mathrm{~mm}$-sodium acetate buffer, $\mathrm{pH} 5.0$, at $37^{\circ} \mathrm{C}$ for $20 \mathrm{~min}$. Finally, the sections were washed under running tap water and mounted with Aquamount (Gurr, Hopkin \& Williams, Chadwell Heath, U.K.) and microscopically examined. The following controls were included: the anti-relaxin antiserum was replaced by the same antiserum after absorption with purified porcine relaxin $(167 \mu \mathrm{g} / \mathrm{ml})$, or by normal rabbit serum, or by omitting the first or the second antiserum in the staining procedure.

\section{Results}

In double diffusion, a single precipitation line was observed between purified porcine relaxin and anti-porcine relaxin antiserum. Sera from pregnant women, human prolactin, human placental lactogen, human growth hormone, porcine insulin, hCG and SPl caused no precipitation.

Specific relaxin immunoreactivity was seen in the glandular epithelium of the prostate in all the specimens stained with the NIH-RXN-P1-3000 antiserum or the R6 antiserum. The staining was unevenly distributed, some areas being more strongly stained than others (Pl. 1, Figs 1-3). In the seminal vesicles and in the ampullary part of the vas deferens, the staining was evenly distributed in the glandular epithelium (Pl. 2, Figs 4-6). Similar results were obtained with both antisera. Relaxin was not detected in the testis or epididymis. 


\section{PLATE 1}
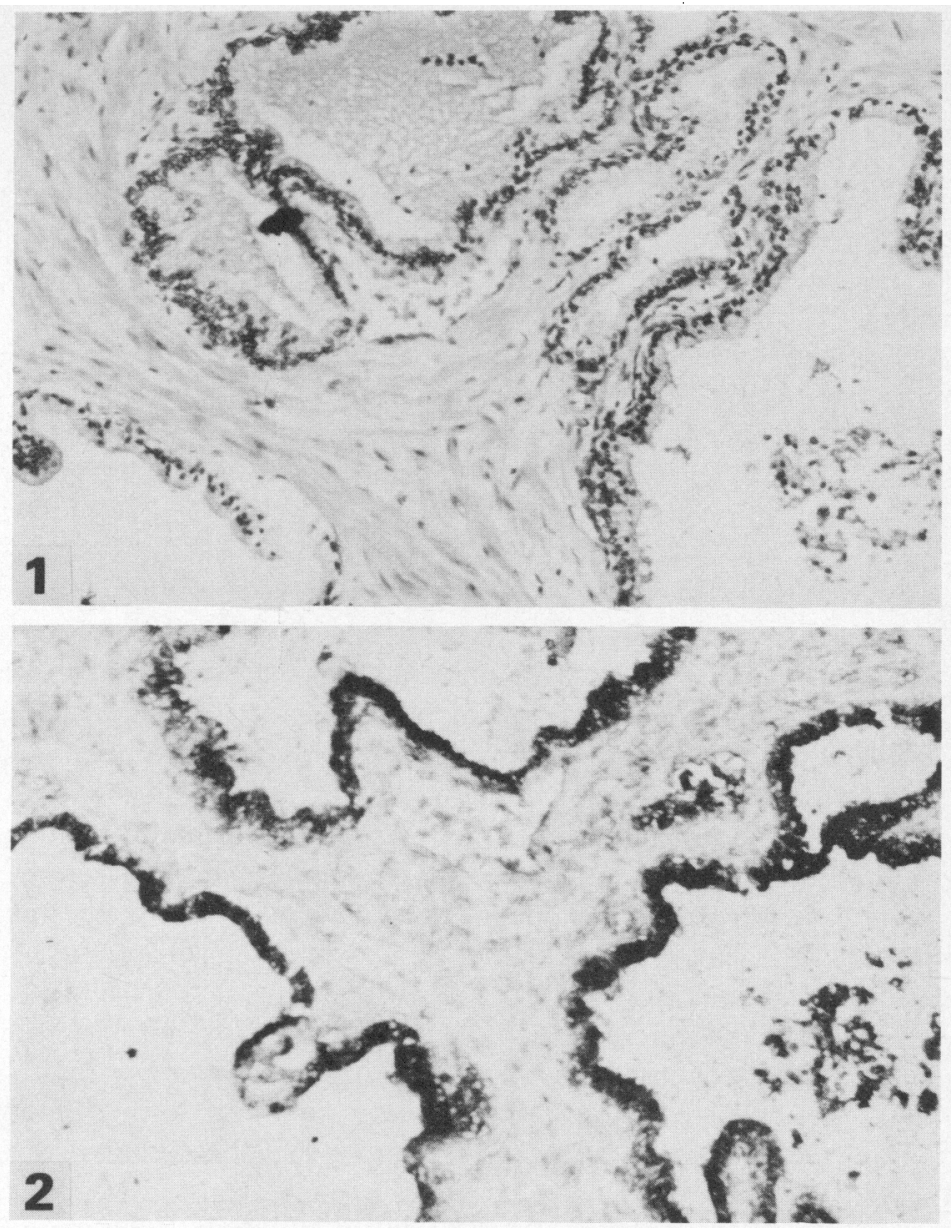

\section{3}

Figs 1-3. Immunohistochemical demonstration of relaxin in the prostate gland $\times 380$.

Fig. 1. Haematoxylin-eosin staining.

Fig. 2. Anti-relaxin antiserum (NIH-RXN-P1-3000 antiserum). The cells of the glandular epithelium are positive .

Fig. 3. Adjacent control section stained with anti-relaxin antiserum absorbed with relaxin. 
PLATE 2
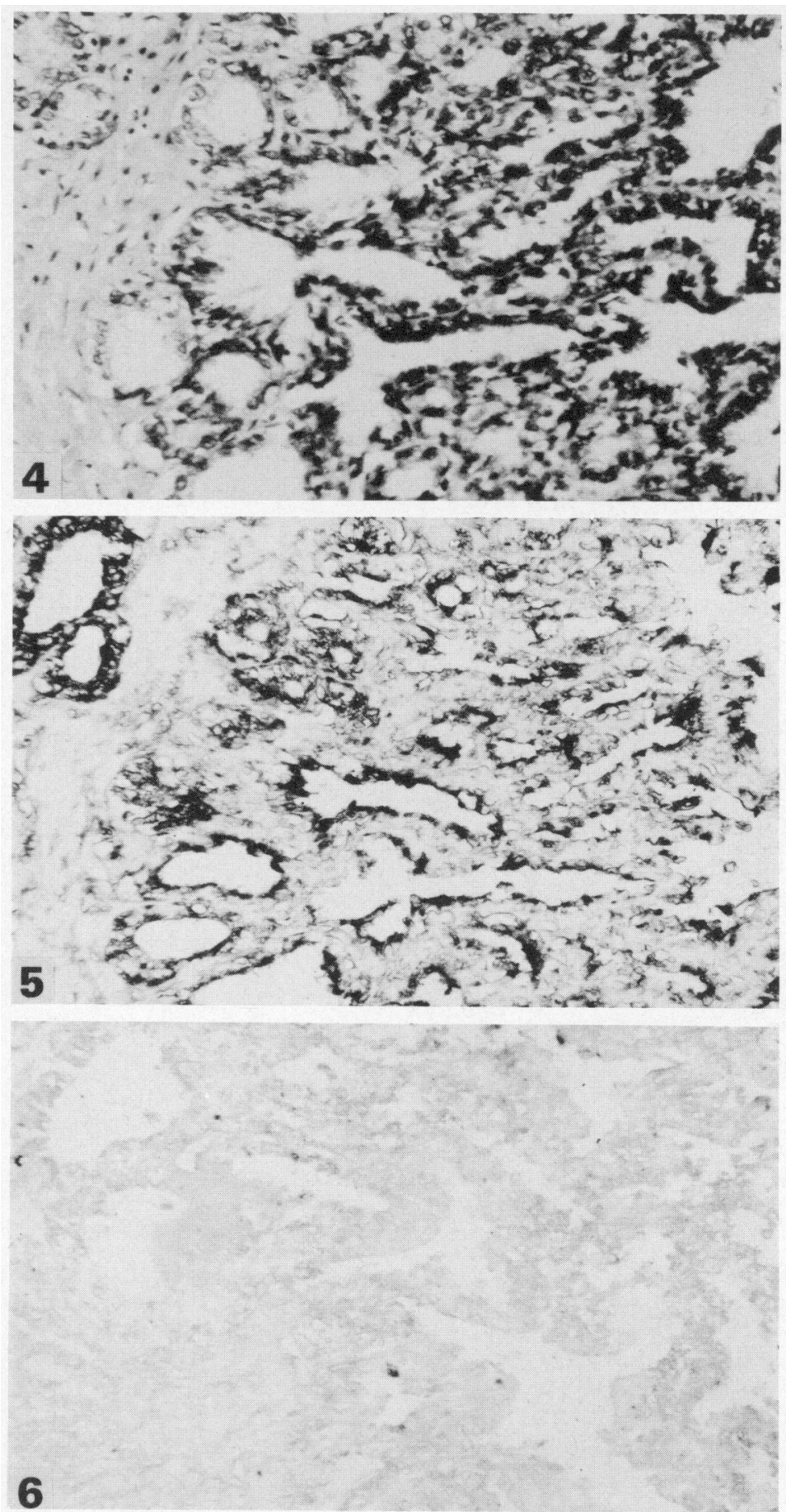

Figs 4-6. Immunoperoxidase staining of relaxin in the seminal vesicle. $\times 380$.

Fig. 4. Haematoxylin-eosin staining.

Fig. 5. Anti-relaxin antiserum (NIH-RXN-P1-3000 antiserum). The cells of the glandular epithelium are positive.

Fig. 6. Adjacent control section stained with anti-relaxin antiserum absorbed with relaxin. 


\section{Discussion}

Our results show the localization of relaxin in the male genital tract. Relaxin was present in the glandular epithelium of the prostate and also in the seminal vesicles and the ampullary part of the vas deferens. When assessing the validity of our results, attention must be paid to the purity of the immunizing antigen and the specificity of the antisera. The R6 antiserum against highly purified porcine relaxin cross-reacts with relaxin from the human seminal plasma (Loumaye et al., 1980). Therefore, it is not surprising that the antiserum we prepared against highly purified relaxin (NIHRXN-P1, $3000 \mathrm{U} / \mathrm{mg}$ ) reacts with male tissues. Reduction of staining was observed following absorption with NIH-RXN-P1, $3000 \mathrm{U} / \mathrm{mg}$, the relaxin preparation now recommended as the standard preparation for bioassays (Bryant-Greenwood, 1982). Cameron et al. (1982) used the same antigen to absorb their antiserum before control stainings when they reported the occurrence of relaxin in the armadillo prostate gland. Dubois \& Dacheux (1978), using antiserum prepared against crude porcine relaxin (NIH porcine relaxin, $442 \mathrm{U} / \mathrm{mg}$ ) coupled to human albumin, detected relaxin-like immunoreactivity in the testis of the adult boar. However, Arakaki et al. (1980) were unable to repeat this work with an antiserum raised against highly purified relaxin, and our negative results on the human testis are consistent with this.

Our results suggest that relaxin is either synthesized or taken up by cells in the prostate and the seminal vesicles. Relaxin has been shown to increase sperm motility (Essig et al., 1982) and, in the female, relaxin induces cervical ripening (MacLennan, Green, Bryant-Greenwood, Greenwood \& Seamark, 1980). It seems possible that human seminal plasma relaxin, secreted by the glandular epithelium of the prostate, seminal vesicle and vas deferens, exerts a local effect on the cervix and facilitates the passage of spermatozoa in the female genital tract.

This study was supported by the Finnish Cultural Foundation (H.Y.-J.), the Cancer Society of Finland (T.W.) and the Sigrid Juselius Foundation, and the Research Council for Medical Sciences, Academy of Finland (M.S.). We thank Ms Tuula Halmesvaara for technical assistance.

\section{References}

Arakaki, R.F., Kleifeld, R.G. \& Bryant-Greenwood, G.D. (1980) Immunofluorescence studies using antisera to crude and to purified porcine relaxin. Biol. Reprod. 23, $153-159$.

Bryant-Greenwood, G.D. (1982) Relaxin as a new hormone. Endocrine Reviews 3, 62-90.

Cameron, D.F., Corton, G.L. \& Larkin, L.H. (1982) Relaxin-like antigenicity in the armadillo prostate gland. Ann. N.Y. Acad. Sci. 380, 231-240.

Dubois, M.P. \& Dacheux, J.L. (1978) Relaxin, a male hormone? Cell Tiss. Res. 187, 201-214.

Essig, M., Schoenfeld, C., D'Eletto, R., Amelar, R., Dubin, L., Steinetz, B., O'Byrne, E. \& Weiss, G. (1981) Relaxin in human seminal plasma. Ann. N.Y. Acad. Sci. 380, 224-230.

Loumaye, E., Teuwissen, B. \& Thomas, K. (1978) Characterization of relaxin radioimmunoassay using Bolton-Hunter reagent. Gynecol. Obstet. Invest. 9, $262-267$.

Loumaye, E., de Cooman, S. \& Thomas, K. (1980) Immunoreactive relaxin-like substance in human seminal plasma. J. clin. Endocr. Metab. 50, 11421143.

MacLennan, A.H., Green, R.C., Bryant-Greenwood, G.D., Greenwood, F.C. \& Seamark, R.F. (1980) Ripening of the human cervix and induction of labour with purified porcine relaxin. Lancet i, 220223.

O'Byrne, E.M. \& Steinetz, B.G. (1976) Radioimmunoassay (RIA) of relaxin in sera of various species using an antiserum to porcine relaxin. Proc. Soc. exp. Biol. Med. 152, 272-276.

O'Byrne, E.M., Carriere, B.T., Sorensen, L., Segaloff, A. \& Schwabe, C. (1978a) Plasma immunoreactive relaxin levels in pregnant and nonpregnant women. J. clin. Endocr. Metab. 47, 1106-1110.

O'Byrne, E.M., Flitcraft, J.F., Sawyer, W.K., Hochman, J., Weiss, G. \& Steinetz, B.G. (1978b) Relaxin bioactivity and immunoactivity in human corpora Jutea. Endocrinology 102, 1641-1644.

Quagliarello, J., Szlachter, N., Steinetz, B.G., Goldsmith, L.T. \& Weiss, G. (1979) Serial relaxin concentrations in human pregnancy. Am. J. Obstet. Gynec. 135, 4344.

Sherwood, O.D. \& O'Byrme, E.M. (1974) Purification and characterization of porcine relaxin. Archs Biochem. Biophys. 160, 185-196.

Steinetz, B.G., O'Byrne, E.M., Weiss, G. \& Schwabe, C. (1979) Bioassay methods for relaxin: uses and pitfalls. In Relaxin, pp. 79-113. Ed. R. R. Anderson. Plenum, New York. 